\title{
L-PGDS (Betatrace Protein) Inhibits Astrocyte Proliferation and Mitochondrial ATP Production in Vitro
}

\author{
Xiaorong Xin • Andreas Huber • Peter Meyer • \\ Josef Flammer • Albert Neutzner • Neil R. Miller • \\ Hanspeter E. Killer
}

Received: 5 June 2009 / Accepted: 29 June 2009 /Published online: 14 July 2009

(C) Humana Press 2009

\begin{abstract}
L-PGDS is the most abundant protein present in the cerebrospinal fluid (CSF). Although CSF was believed to be homogenous in content, a previous study has showed that a marked concentration gradient of L-PGDS exists between the spinal CSF and the CSF in the subarachnoid space of patients with optic nerve disease (papilledema and normaltension glaucoma). Astrocytes play a critical role in maintaining the integrity of axon function in the central nervous system and specifically in the optic nerve, and we therefore investigated the biochemical effects of L-PGDS on the proliferation of astrocytes and on the production of adenosine triphosphate (ATP) by astrocyte mitochondria. We found an inhibitory effect of L-PGDS on both proliferation of astrocytes and production of astrocyte ATP. The concentrations that inhibited astrocyte proliferation and ATP production were in the range measured in patients with
\end{abstract}

Hanspeter E. Killer MD is the corresponding author of this paper.

X. Xin · P. Meyer · J. Flammer • A. Neutzner $\cdot$ H. E. Killer University Eye Clinic,

Basel, Switzerland

\section{A. Huber}

Department of Laboratory Medicine, Kantonsspital Aarau, Aarau, Switzerland

H. E. Killer $(\bowtie)$

Department of Ophthalmology, Kantonsspital Aarau,

Aarau, Switzerland

e-mail: Killer@ksa.ch

X. Xin

Department of Ophthalmology,

Qinghai Provincial People's Hospital,

Xining, Qinghai, China

N. R. Miller

Wilmer Eye Institute, Johns Hopkins Hospital,

Baltimore, MD, USA idiopathic intracranial hypertension and in patients with normal-tension glaucoma. As the CSF is in contact with axons and mitochondria of the optic nerve (Bristow et al. Archives of Ophthalmology, 120, 791-796, 2002), we postulate that a change in the concentration of CSF protein such as L-PGDS could exercise a harmful effect on these structures.

Keywords Betatrace protein - L-PGDS · Astrocytes . Mitochondria $\cdot$ Optic nerve $\cdot$ Glaucoma

\section{Introduction}

L-PGDS, also called betatrace protein, is a prostaglandin synthase that consists of 165 amino acid sequences, has a molecular weight of $25 \mathrm{kDa}$, and catalyzes the isomerization of the 9, 11-endoperoxide group of $\mathrm{PGH}_{2}$, a precursor of various protanoids, to produce $\mathrm{PGD}_{2}$ with 9-hydroxy and 11-keto groups (Clausen 1961; Hoffmann et al. 1993, 1996; Urade and Hayaishi 2000a, b). Although L-PGDS is present in very low concentrations in the serum, it is the most abundant polypeptide synthesized intrathecally (Pohl et al. 1997; Urade and Hayaishi 2000a). Normal values for L-PGDS in the cerebrospinal fluid (CSF) of healthy persons range from 13.4 to $18.4 \mathrm{mg} / \mathrm{L}$, with a slight increase in concentration with increasing age (Link and Olsson 1972; Tumani et al. 1998; Reiber et al. 2003).

L-PGDS mRNA was first detected in mesenchymal cells destined to become leptomeninges and in developing testis (Tokugawa et al. 1998). Immunoreactivity and mRNA for L-PGDS were subsequently identified in meningoepithelial cells, arachnoid trabecular cells, and arachnoid pia mater cells as well as in oligodendrocytes and astrocytes (Urade et al. 1993; Blödorn et al. 1999; Beuckmann et al. 2000; Urade and Hayaishi 2000a, b), and it eventually became 
clear that L-PGDS is localized primarily in the central nervous system (CNS) and related organs, such as the cochlea and the eye (e.g., in the pigment and ciliary body epithelium) (Goh et al. 1987), where the production and secretion of L-PGDS has been demonstrated in cultured astrocytes, leptomeningeal cells, and choroid plexus epithelium (Giacomelli et al. 1996).

L-PGDS belongs to the family of lipocalins, a group of proteins responsible for binding and transporting small lipophilic molecules, such as bilirubin, biliverdin, retinol, and sexual pheromones (Beuckmann et al. 2000; Urade and Hayaishi 2000a). L-PGDS concentration in rat CSF shows a circadian change coupled to the sleep rhythm, with the concentration increasing with increased sleep (Hayaishi et al. 1991).

Some of the functions of L-PGDS seem contradictory. Specifically, although increased L-PGDS activity appears to correlate with apoptosis of PC12 neuronal cells as well as phorbol 12-myristate, 13-acetate (PMA)-induced apoptosis (Ragolia et al. 2001, 2003), upregulated L-PGDS also has been demonstrated in $\alpha \beta$-crystallin-positive oligodendrocytes and astrocytes in patients with chronic multiple sclerosis in whom it is believed to play a role in neuroprotection (Kagitani-Shimono et al. 2006). In addition, in an animal model, Liang et al (2005) described a neuroprotective effect of hippocampal neurons via $\mathrm{PGD}_{2}$ (L-PGDS) mediated by the DP1 receptor. Elevated concentrations of betatrace protein in CSF have been found in patients with acute exacerbations of multiple sclerosis (Link and Olsson 1972). Upregulation of L-PGDS was also demonstrated in oligodendrocytes after hypoxic-ischemic injury in developing brains in mouse models and in the brains of patients with multiple sclerosis (Taniguchi et al. 2007a). Based on these findings, Taniguchi and colleagues (Taniguchi et al. 2007a, b) suggest that L-PGDS may function as an early stress protein with immunomodulatory and neuroprotective activity. These findings may explain the fact that high levels of L-PGDS are present in the subarachnoid space (SAS) of the optic nerve in patients with idiopathic intracranial hypertension (IIH) and normal-tension glaucoma (NTG) (Killer et al. 2006, 2007). In addition, a marked concentration gradient of L-PGDS exists between the spinal CSF and the CSF in the SAS of the optic nerves in such patients (Killer et al. 2006, 2007). At issue is whether the findings are related to the development of these conditions or simply represent an epiphenomena, i.e., is there a biological effect of L-PGDS that could account for either the development of the conditions or the optic nerve damage that occurs from them? As astrocyte function and adenosine triphosphate (ATP) production are important for axonal function, especially for white-matter axonal regeneration (Tom et al., 2004; Butt et al. 2004), we investigated the biochemical effects of L-PGDS on the proliferation of astrocytes and on the production of ATP by astrocyte mitochondria in vitro.

\section{Materials and Methods}

\section{Cell Culture and Treatment with L-PGDS}

The astrocyte cell line LN 229 (courtesy of Dr. Stephan Frank, Department of Pathology, University of Basel, Switzerland) was grown in $75-\mathrm{cm}^{2}$ flasks (Sarstedt, USA) and cultured in Quantum 263 medium (PAA Biologics GmbH, Cöllbe, Germany) with penicillin/streptomycin (100 U/mL, $100 \mu \mathrm{g} / \mathrm{mL}$; Sigma, Germany) until confluence. Cells were washed with phosphate-buffered saline (PBS) (Sigma) and trypsinized. Supernatant was removed after centrifugation, and cell suspension was added to sixwell plates (Falcon, USA) pre-coated with collagen (Sigma), with each well containing $2 \times 10^{4}$ cells. The medium was replaced by fresh culture medium containing human L-PGDS (courtesy of Prof. H.S. Conradt; GlycoThera GmbH, Feodor-Lynen-Straße 35, 30625 Hannover, Niedersachsen, Germany) with series concentration $(7,14$, $35,70,140 \mu \mathrm{g} / \mathrm{mL}$ ) or no agonist ( 0 as control).

\section{Proliferation Assay}

After incubation with L-PGDS for $72 \mathrm{~h}$, cells were washed with PBS without $\mathrm{Ca}^{2+}$ or $\mathrm{Mg}^{2+}$ and then harvested. Cell number, cell diameter, and cell volume were determined by a cell analyzer system CASY1 (Schaerfe System, Reutlingen, Germany). Samples of suspended cells $(100 \mu \mathrm{L})$ were diluted in $10 \mathrm{~mL}$ isotonic CASY solution $(7.93 \mathrm{mg} / \mathrm{mL} \mathrm{NaCl}$, $0.38 \mathrm{mg} / \mathrm{mL} \mathrm{Na} \mathrm{Na}_{2}$ EDTA, $0.4 \mathrm{mg} / \mathrm{mL} \mathrm{KCl}, 0.22 \mathrm{mg} / \mathrm{mL}$ $\mathrm{NaH}_{2} \mathrm{PO}_{4} \cdot \mathrm{H}_{2} \mathrm{O}, 2.45 \mathrm{mg} / \mathrm{mL} \quad \mathrm{Na}_{2} \mathrm{HPO} \cdot 2 \mathrm{H}_{2} \mathrm{O}, 0.3 \mathrm{mg} / \mathrm{mL}$ $\mathrm{NaF}$; Schaerfe System). During the measurement, a current was supplied to the capillary via two platinum electrodes giving a defined electrical resistance. Thus, while passing the capillary, the cells generated electrical signals and could be scanned, providing data regarding cell number, diameter, and volume (Stadlmann et al. 2006).

\section{ATP Determination}

Fifty thousand cells per well were seeded into 12-well plates (Falcon, USA), cultured with Quantum 263 medium containing L-PGDS $70 \mu \mathrm{g} / \mathrm{mL}$ or $10 \%$ PBS for 1 and 2 days, respectively. Culture medium was removed and ATP incubation medium (KCL $15 \mathrm{mM}$, Tris- $\mathrm{HCl} 25 \mathrm{mM}$, EDTA $0.2 \mathrm{mM}, \mathrm{KH}_{2} \mathrm{PO}_{4} 1 \mathrm{mM}, \mathrm{pH} 7.4,1 \%$ albumin, ADP $1 \mathrm{mM}$ ) with or without pyruvate $100 \mathrm{mM}$ and malate $100 \mathrm{mM}$ was added, and the cells were incubated at $37^{\circ} \mathrm{C}$ for $2 \mathrm{~h}$. After incubation medium was withdrawn and cells were washed with PBS, incubation medium without ADP was added, and the cells were detached with a cell scraper. Cell supernatant was collected into a micro test tube, boiled with Tris-EDTA, and centrifuged at $1,000 \times g$ at room 


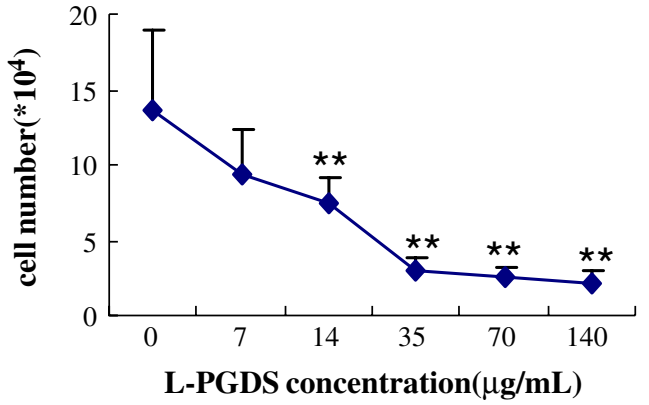

Fig. 1 Effect of L-PGDS on astrocytes proliferation. Proliferation of astrocytes decreased with an increase of the concentration of L-PGDS. Evident decrease of cell number was detected at L-PGDS concentration ranging from 14 to $140 \mu \mathrm{g} / \mathrm{mL}$. Results are mean \pm SD of six experiments. Statistically different data are indicated by asterisks, ** represent $p<0.005$ compared with controls

temperature for $1 \mathrm{~min}$. The supernatant was then collected for protein and ATP assay.

The total protein concentration in each sample was analyzed by Bradford protein (BIO-RAD, Germany) assay with a spectrophotometer (Spectra MAX 190, Bucher Biotec, USA). Samples were placed in a 96-well plate (Costar, USA), and BSA protein (Sigma, USA) with series concentration $(0-0.2 \mathrm{mg} / \mathrm{mL})$ was utilized as the standard to normalize the assay. Optical absorption at $595 \mathrm{~nm}$ was measured. The quantity of ATP was measured by using an ATP determination kit (A-22066, Eugene, OR, USA) following the manufacturer's instructions. The reagents and reaction mixture were combined according to the protocol by molecular probes. Two microliters of the sample and $198 \mu \mathrm{L}$ of the reagent of the ATP determination kit were placed into 96-well assay microplates and reacted to quantify ATP production. Luminescence was measured using a luminometer (Berthold Technologies GmbH, Switzerland), and ATP production was quantified using a standard curve from 0 to $50 \mu \mathrm{M}$. ATP production was expressed as $\mu \mathrm{M} / \mu \mathrm{g}$ mitochondrial protein (Drew and Leeuwenburgh 2003).

\section{Statistical Analysis}

For astrocyte proliferation analysis, one-way analysis of variance followed by post hoc comparisons of the group means according to the method of Bonferroni was used. For ATP determination, data were analyzed with three-way analysis of variance, and the Student's $t$ test was performed to ascertain the statistical significance between two experiments treated with the same reagent at different times. Statistical analyses were conducted with SPSS 10.0 statistical analysis software (SPSS Inc., Chicago, IL, USA), with data presented as mean \pm SD. Values of $p<0.05$ were considered significant.

\section{Results}

\section{Inhibition of Proliferation of Astrocytes}

Incubation of astrocytes with L-PGDS for $72 \mathrm{~h}$ resulted in an inhibition of cellular proliferation that correlated with the concentration of L-PGDS (Fig. 1). Specifically, treatment of
Fig. 2 Effect of L-PGDS on proliferation of astrocytes. a Astrocytes of controls after the incubation for 1 day. b Astrocytes treated with L-PGDS $(70 \mu \mathrm{g} / \mathrm{mL})$ after incubation for 1 day. $\mathbf{c}$ Obvious proliferation of astrocytes of controls is observed after incubation for 3 days. d Number of astrocytes treated with L-PGDS $(70 \mu \mathrm{g} / \mathrm{mL})$ after incubation for 3 days is much less than that of controls incubated for the same period $(\times 10)$
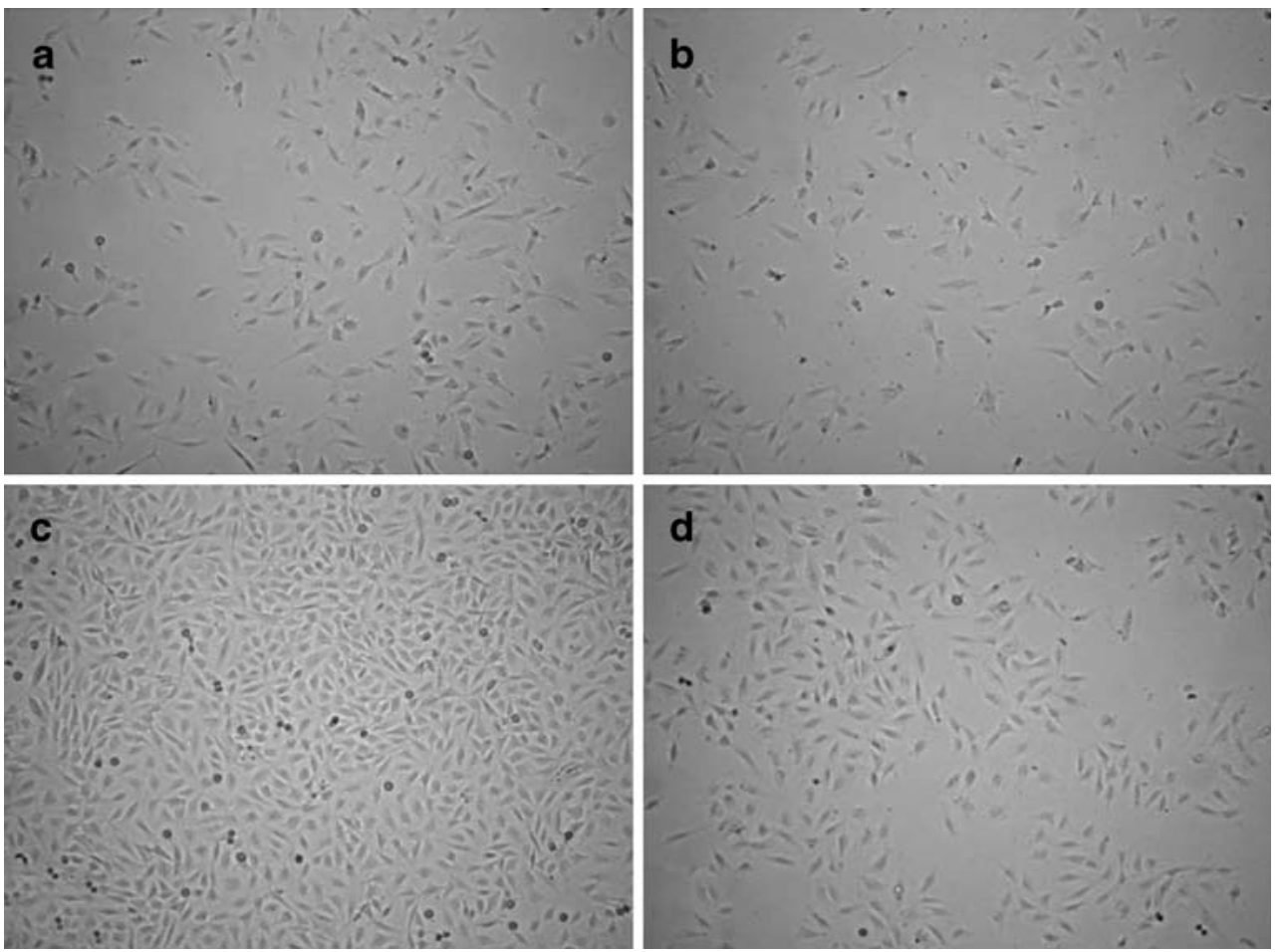
Table 1 Cell number, diameter, and volume of astrocytes (LN 229) incubated with L-PGDS as determined by CASY

\begin{tabular}{lrcc}
\hline L-PGDS concentration $(\mu \mathrm{g} / \mathrm{mL})$ & Cell number $\left(10^{4}\right)$ & Cell diameter $(\mu \mathrm{m})$ & Cell volume $\left(10^{3} \mathrm{fL}\right)$ \\
\hline 0 & $13.597 \pm 5.248$ & $18.635 \pm 0.608$ & $3.849 \pm 0.528$ \\
7 & $9.320 \pm 3.051$ & $18.445 \pm 0.353$ & $3.707 \pm 0.217$ \\
14 & $7.357 \pm 1.860$ & $18.343 \pm 0.281$ & $3.639 \pm 0.171$ \\
35 & $3.042 \pm 0.860$ & $18.297 \pm 0.550$ & $3.792 \pm 0.319$ \\
70 & $2.490 \pm 0.630$ & $18.327 \pm 0.685$ & $3.661 \pm 0.326$ \\
140 & $2.189 \pm 0.791$ & $18.007 \pm 0.473$ & $3.693 \pm 0.581$ \\
\hline
\end{tabular}

astrocytes with L-PGDS at a dose of $14 \mu \mathrm{g} / \mathrm{mL}$ resulted in a decrease in cell number $(73,573 \pm 18,600)$ compared with controls $(135,970 \pm 52,480 ; p<0.005)$, with the number of cells decreasing with increasing concentrations of L-PGDS of 70 and $140 \mu \mathrm{g} / \mathrm{mL}$. In our cultures, L-PGDS produced its maximal inhibitory effect at a dose of $140 \mu \mathrm{g} / \mathrm{mL}$, resulting in a cell number of $21,890 \pm 7,910(p<0.005)$, decreased sixfold compared with controls. As shown in Fig. 2, astrocytes from controls showed extensive proliferation after an incubation time of 3 days compared with those incubated for 1 day (Fig. 2a, c); the proliferation of cells treated with L-PGDS at a dose of $70 \mu \mathrm{g} / \mathrm{mL}$, however, was inhibited (Fig. 2b, d). The diameter of astrocytes after treatment with L-PGDS as determined by CASY I decreased from $18.445 \pm$ 0.353 to $18.007 \pm 0.473 \mu \mathrm{m}$ with concentrations of L-PGDS ranging from 7 to $140 \mu \mathrm{g} / \mathrm{mL}$, with the maximum reduction (18.007 $\pm 0.473 \mu \mathrm{m}, p=0.625)$ occurring with a dose of $140 \mu \mathrm{g} / \mathrm{mL}$. There was no significant difference in astrocyte mean cell volume with increasing concentrations of L-PGDS compared with controls $(3,792 \pm 319 \mathrm{fL}$ to $3,661 \pm 326 \mathrm{fL}$ vs. $3,849 \pm 528 \mathrm{fL} ; p=0.93$ ) (Table 1).

\section{Effect of L-PGDS on ATP Production}

Cellular ATP production was measured after astrocytes were treated with PBS (controls), L-PGDS, mitochondrial com-

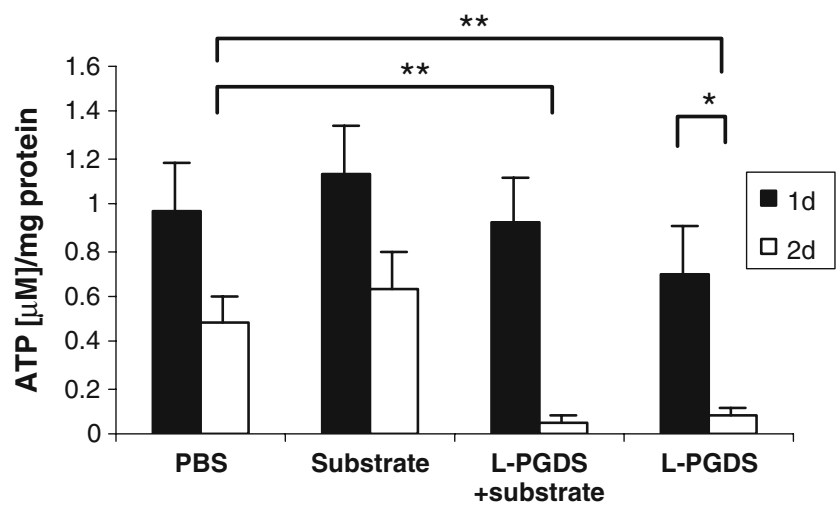

Fig. 3 Effect of L-PGDS on ATP production of astrocytes. L-PGDS $(70 \mu \mathrm{g} / \mathrm{mL})$ showed an inhibitory effect on ATP production after incubation for 2 days. Data are represented as mean \pm SD of four independent experiments. ${ }^{*} p<0.05 ;{ }^{* *} p<0.01$ plex I substrates (pyruvate + malate), or L-PGDS plus substrates for 1 and 2 days. After 1 day, there was an increase in ATP production in cells incubated with either PBS or substrate without L-PGDS compared with cells treated with L-PGDS; however, none of the changes in ATP production with substrate $(1.135 \pm 0.582 \mu \mathrm{M} / \mathrm{mg}$ protein), L-PGDS $(0.698 \pm 0.246 \mu \mathrm{M} / \mathrm{mg}$ protein $)$, or L-PGDS plus substrate $(0.914 \pm 0.547 \mu \mathrm{M} / \mathrm{mg}$ protein $)$ were significantly different from controls $(0.972 \pm 0.396 \mu \mathrm{M} / \mathrm{mg}$ protein, $p>$ 0.05) (Fig. 3). After 2 days of incubation with either PBS or substrate alone, ATP production was unchanged compared with ATP production after 1 day of incubation (Fig. 3), whereas cells treated with L-PGDS with or without substrate showed a marked decrease in ATP production $(0.056 \pm 0.019$, $0.074 \pm 0.036 \mu \mathrm{M} / \mathrm{mg}$ protein, respectively) compared with cells incubated with PBS $(0.492 \pm 0.099 \mu \mathrm{M} / \mathrm{mg}$ protein $)$ or substrate alone $(0.633 \pm 0.159 \mu \mathrm{M} / \mathrm{mg}$ protein $)$ for the same length of time $(p<0.01)$, indicating an inhibitory effect by L-PGDS. In addition, cells incubated with L-PGDS for 2 days showed significantly reduced ATP production compared with cells incubated with L-PGDS for 1 day $(p<$ 0.05 ) (Fig. 3). The effect of L-PGDS and of incubation time on ATP production was highly significant $(p=0.002, p<$ 0.001 , respectively).

\section{Discussion}

In this study, we report that L-PGDS inhibits the proliferation of astrocytes in cell culture, with the degree of inhibition correlating with the concentration of L-PGDS. In addition, astrocytes incubated with L-PGDS show reduced ATP production, with the amount of reduction increasing with an increased incubation time. Thus, it appears that L-PGDS suppresses mitochondrial function in astrocytes regardless of the availability of mitochondrial complex I substrates malate and pyruvate.

The decline of the astrocyte cell number correlates with an increase of L-PGDS concentration and also with an increased time of incubation, particularly when the L-PGDS concentration exceeds the maximum normal physiological concentration $140 \mu \mathrm{g} / \mathrm{mL}$ (Fig. 1). Indeed, when astrocytes are incubated with L-PGDS in a concen- 
tration of $70 \mu \mathrm{g} / \mathrm{mL}$ (a concentration found in the CSF of the subarachnoid space of the optic nerve in some patients with IIH (Killer et al. 2007)), there is a significant reduction of ATP production after 2 days of incubation (Fig. 3).

It is clear from our study that L-PGDS both inhibits proliferation of astrocytes and reduces production of ATP by these cells. The mechanism for these effects is not known and there are several possible mechanisms by which these effects could occur. For example, L-PGDS has the potential to uncouple the oxidative phosphorylation chain in mitochondria (Kanekiyo et al. 2007), thus decreasing ATP production. This, in turn, could lead to reduced growth of astrocytes. Such an effect might be enhanced by the chaperone activity of L-PGDS (Kanekiyo et al. 2007). In a recent article, Xiang et al. (2007) reported that PGD2 and 15d-PGJ2 induced apoptosis of undifferentiated oligodendrocytes. L-PGDS is involved in the enzymatic process producing these molecules and thus might play a role in the process of myelin damage.

Astrocytes are among the most robust cells in the CNS. The inhibition of their proliferation by L-PGDS reflects the high biological activity of this substance. Thus, it seems possible that less robust structures such as the axons and their myelin sheaths might be even more susceptible to damage caused by elevated L-PGDS concentrations. Finally, high concentrations of L-PGDS might also be toxic to the astrocytes.

Regardless of the mechanism by which L-PGDS inhibits astrocyte metabolism and growth, our findings are consistent with the results of other investigators that suggest a role for this substance in neurological and ocular disorders that involve glial-neuronal interactions. For example, Kanekiyo et al. found evidence that L-PGDS may act as a major endogenous $\mathrm{A}_{\beta}$ chaperone in the brain and may have a role in the pathogenesis of Alzheimer's disease (Kanekiyo et al. 2007).

In summary, this report describes a significant inhibitory effect of L-PGDS on both proliferation and ATP production of astrocytes in vitro. The concentrations used for treating astrocyte cultures in this study are in the range of concentrations measured in patients with disorders that are associated with optic nerve damage, such as IIH and NTG. The pathophysiology of NTG is not yet fully understood; neither is vision loss in patients with IIH. Further studies that evaluate the effect of L-PGDS on glial cells in the optic nerve are needed to define the role of elevated L-PGDS concentration in optic nerve disease.

Acknowledgements We would like to thank Harald S. Conradt MD from GlycoThera $\mathrm{GmbH}$ for providing the $\beta$-trace protein used in this study and the De Bernardi Fond Basel, the Horton Foundation, the Emilia Guggenheim-Schnurr Foundation, and the Herta Messerli Foundation.

\section{References}

Beuckmann, C. T., Lazarus, M., Gerashchenko, D., et al. (2000). Cellular localization of lipocalin-type prostaglandin D synthase (beta-trace) in the central nervous system of the adult rat. Journal of Comparative Neurology, 428, 62-78.

Blödorn, B., Brück, W., Tumani, H., et al. (1999). Expression of the beta-trace protein in human pachymeninx as revealed by in situ hybridization and immunocytochemistry. Journal of Neuroscience Research, 57, 730-734.

Bristow, E. A., Griffiths, P. G., Andrews, R. M., Johnson, M. A., \& Turnbull, D. M. (2002). The distribution of mitochondrial activity in relation to optic nerve structure. Archives of Ophthalmology, 120, 791-796.

Butt, A. M., Pugh, M., Hubbard, P., \& James, G. (2004). Functions of optic nerve glia: axoglial signalling in physiology and pathology. Eye, 18, 1110-1121.

Clausen, J. (1961). Proteins in normal cerebrospinal fluid not found in serum. Proceedings of the Society for Experimental Biology and Medicine, 107, 170-172.

Drew, B., \& Leeuwenburgh, C. (2003). Method for measuring ATP production in isolated mitochondria: ATP production in brain and liver mitochondria of Fischer-344 rats with age and caloric restriction. American Journal of Physiology-Regulatory, Integrative and Comparative Physiology, 285, R1259-R1267.

Giacomelli, S., Leone, M. G., Grima, J., Silvestrini, B., \& Cheng, C. Y. (1996). Astrocytes synthesize and secrete prostaglandin D synthetase in vitro. Biochimica et Biophysica Acta-Molecular Cell Research, 1310, 269-276.

Goh, Y., Urade, Y., Fujimoto, N., \& Hayaishi, O. (1987). Content and formation of prostaglandins and distribution of prostaglandinrelated enzyme activities in the rat ocular system. Biochimica et Biophysica Acta, 921, 302-311.

Hayaishi, O., Matsumura, H., Onoe, H., Koyama, Y., \& Watanabe, Y. (1991). Sleep-wake regulation by PGD2 and E2. Advances in Prostaglandin, Thromboxane, and Leukotriene Research, 21B, 723-726.

Hoffmann, A., Conradt, H. S., Gross, G., Nimtz, M., Lottspeich, F., \& Wurster, U. (1993). Purification and chemical characterization of beta-trace protein from human cerebrospinal fluid: its identification as prostaglandin D synthase. Journal of Neurochemistry, 61, 451-456.

Hoffmann, A., Bächner, D., Betat, N., Lauber, J., \& Gross, G. (1996). Developmental expression of murine Beta-trace in embryos and adult animals suggests a function in maturation and maintenance of blood-tissue barriers. Developmental Dynamics, 207, 332-343.

Kagitani-Shimono, K., Mohri, I., Oda, H., et al. (2006). Lipocalin-type prostaglandin D synthase (beta-trace) is upregulated in the alphaB-crystallin-positive oligodendrocytes and astrocytes in the chronic multiple sclerosis. Neuropathology and Applied Neurobiology, 32, 64-73.

Kanekiyo, T., Ban, T., Aritake, K., et al. (2007). Lipocalin-type prostaglandin D synthase/-trace is a major amyloid betachaperone in human cerebrospinal fluid. Proceedings of the National Academy of Sciences of the United States of America, 104, 6412-6417.

Killer, H. E., Jaggi, G. P., Flammer, J., Miller, N. R., \& Huber, A. R. (2006). The optic nerve: a new window into cerebrospinal fluid composition? Brain, 129, 1027-1030.

Killer, H. E., Jaggi, G. P., Flammer, J., Miller, N. R., Huber, A. R., \& Mironov, A. (2007). Cerebrospinal fluid dynamics between the intracranial and the subarachnoid space of the optic nerve. Is it always bidirectional? Brain, 130, 514-520.

Liang, X., Wu, L., Hand, T., \& Andreasson, K. (2005). Prostaglandin D2 mediates neuronal protection via the DP1 receptor. Journal of Neurochemistry, 92, 477-486. 
Link, H., \& Olsson, J. E. (1972). Beta-trace protein concentration in CSF in neurological disorders. Acta Neurol. Scand., 48, 57-68.

Pohl, S., Hoffmann, A., Rüdiger, A., Nimtz, M., Jaeken, J., \& Conradt, H. S. (1997). Hypoglycosylation of a brain glycoprotein ( $\beta$-trace protein) in CDG syndromes due to phosphomannomutase deficiency and $\mathrm{N}$-acetylglucosaminyl-transferase II deficiency. Glycobiology, 7, 1077-1084.

Ragolia, L., Palaia, T., Frese, L., Fishbane, S., \& Maesaka, J. K. (2001). Prostaglandin D2 synthase induces apoptosis in PC12 neuronal cells. NeuroReport, 12, 2623-2628.

Ragolia, L., Palaia, T., Paric, E., \& Maesaka, J. K. (2003). Elevated LPGDS activity contributes to PMA-induced apoptosis concomitant with down regulation of PI3-K. American Journal of Physiology-Cell Physiology, 284, C119-126.

Reiber, H., Walther, K., \& Althaus, H. (2003). Beta-trace protein as sensitive marker for CSF rhinorhea and CSF otorhea. Acta Neurol. Scand., 108, 359-362.

Stadlmann, S., Pollheimer, J., Renner, K., Zeimet, A. G., Offner, F. A., $\&$ Amberger, A. (2006). Response of human peritoneal mesothelial cells to inflammatory injury is regulated by interleukin1beta and tumor necrosis factor-alpha. Wound Repair and Regeneration, 14, 187-194.

Taniguchi, H., Mohri, I., Okabe-Arahori, H., et al. (2007a). Early induction of neuronal lipocalin-type prostaglandin D synthase after hypoxic-ischemic injury in developing brains. Neuroscience Letters, 420, 39-44.
Taniguchi, H., Mohri, I., Okabe-Arahori, H., et al. (2007b). Prostaglandin D2 protects neonatal mouse brain from hypoxic ischemic injury. Journal of Neuroscience, 27, 4303-4312.

Tokugawa, Y., Kunishige, I., Kubota, Y., et al. (1998). Lipocalin-type prostaglandin D synthase in human male reproductive organs and seminal plasma. Biology of Reproduction, 58, 600-607.

Tom, V. J., Doller, C. M., Malouf, A. T., \& Silver, J. (2004). Astrocyteassociated fibronectin is critical for axonal regeneration in adult white matter. Journal of Neuroscience, 24, 9282-9290.

Tumani, H., Nau, R., \& Felgenhauer, K. (1998). Beta-trace protein in cerebrospinal fluid: a blood-CSF barrier-related evaluation in neurological diseases. Annals of Neurology, 44, 882-889.

Urade, Y., Kitahama, K., Ohishi, H., Kaneko, T., Mizuno, N., \& Hayaishi, O. (1993). Dominant expression of mRNA for prostaglandin D synthase in leptomeninges, choroid plexus, and oligodendrocytes of the adult rat brain. Proceedings of the National Academy of Sciences of the United States of America, 90, 9070-9074.

Urade, Y., \& Hayaishi, O. (2000a). Biochemical, structural, genetic, physiological, and pathophysiological features of lipocalin-type prostaglandin D synthase. Biochimica et Biophysica Acta, 1482, 259-271.

Urade, Y., \& Hayaishi, O. (2000b). Prostaglandin D synthase: structure and function. Vitamins and Hormones, 58, 89-120.

Xiang, Z., Lin, T., \& Reeves, S. A. (2007). 15d-PGJ2 induces apoptosis of mouse oligodendrocyte precursor cells. Journal of Neuroinflammation, 4, 18. 